\title{
SIMULTANEOUS SPECTROPHOTOMETRIC DETERMINATION OF NORFLOXACIN, OFLOXACIN AND LOMEFLOXACIN IN RABBIT BLOOD SERUM BY USE OF CHEMOMETRICS
}

\author{
XI-GEN HUANG ${ }^{A *}$, HENG-SONG ZHANG ${ }^{A}$, YAN-XIA LI ${ }^{A}$, MING-FANG LI ${ }^{A}$ \\ ${ }^{a}$ Department of Chemistry, Jiangxi Agricultural University, Nanchang 330045, P. R. China \\ (Received: July 10, 2008 - Accepted: September 16, 2008)
}

\begin{abstract}
A spectrophotometric method for the simultaneous determination of norfloxacin, ofloxacin and lomefloxacin, is described. The spectrophotometric analysis is made possible with the aid of chemometric methods, such as classical least square (CLS), principal component regression (PCR), partial least square (PLS), and radial basis function-artificial neural network (RBF-ANN). Spectra were collected in the 190-400 nm range from a set of samples of three compounds in a Britton-Robinson buffer solution $(\mathrm{pH}=1.81)$. This approach overcomes the significant practical difficulties of severe spectral overlap. It was found that peak intensities are proportional to the concentration for each analyte. The obtained linear calibration concentration ranges are $0.6-13.8,0.5-15.0$, and $0.5-15.0 \mathrm{mg} \mathrm{L}^{-1}$ for norfloxacin, ofloxacin and lomefloxacin, respectively, and the LODs for the three analytes were found to be approximately $6-24 \times 10^{-2} \mathrm{mg} \mathrm{L}^{-1}$. The proposed method was applied to a prediction set of synthetic mixtures of three analytes. The results were shown that the RBF-ANN model gave the best results $\left(\mathrm{RPE}_{\mathrm{T}}=\right.$ $3.0 \%$ ). This method was also applied for the determination of norfloxacin, ofloxacin and lomefloxacin in rabbit blood serum, and these compared well with those from the alternative analysis by HPLC.
\end{abstract}

\section{INTRODUCTION}

Fluoroquinolones are important antibacterial agents developed in the 1980s, and have many applications in veterinary and human medicine. The pharmaceuticals have a broad spectrum of activity and good oral absorption [1]. These drugs are bactericidal over a wide range of therapeutically achievable concentrations, and act via selective inhibition of bacterial DNA synthesis. They have a broad range of action against both Gram negative and Grampositive bacteria $[2,3]$.

In this paper, three important fluoroquinolones, norfloxacin [1-ethyl-6fluoro-1,4-dihydro-4-oxo-7- (1- piperazinyl)-3-quinoline-carboxylic acid], ofloxacin [9-fluoro-2,3-dihydro-3-methyl-10-(4-methyl-1- piperazinyl)-7-oxo7H-pyrido-[1,2,3-de]-1,4-benzoxazine-6-carboxylic acid], and lomefloxacin [1-ethyl-6,8-difluoro-7-(3-methylpiperazin-1-yl)-4-oxoquinoline-3-carboxylic acid] shown in Fig. 1 were investigated and quantitatively analyzed by spectrophotometric method.<smiles>CN1CC(O)C(=O)C2C=C(F)C(=C3CCNCC3)C=C21</smiles>

Norfloxacin

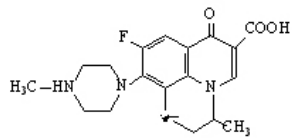

Ofloxacin

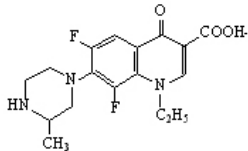

Lomefloxacin
Fig. 1 Structures of norfloxacin, ofloxacin and lomefloxacin.

Although many procedures have been described for the analysis of three fluoroquinolones in pharmaceutical preparations and in biological fluids, reports on the simultaneous determination of the three compounds in these samples are limited to a few methods mainly based on high-performance liquid chromatography $[4,5]$, nuclear magnet resonance [6] and spectrofluorimetry $[7,8]$. However, often these methods are time-consuming $[4,5]$ or require substantial expertise [6] in comparison with spectrophotometry. The spectrophotometric technique is well known, and commonly used for routine analysis. It is simple to apply, has a reasonable sensitivity as well as significant economical advantages [9].

However, to the best of our knowledge, there are no reports on the application of spectrophotometry for the simultaneous determination of the three compounds. This may be explained in the case of traditional spectrophotometry by the structural similarity of the three compounds. Similar UV-vis spectra for the three compounds (Fig. 2) are obtained, and hence, there are significant difficulties for simultaneous determination. A possible solution to this problem is to combine chemometrics with spectrophotometric method. This is known to facilitate the resolution of mixtures of closely related chemical species without any physical separation.

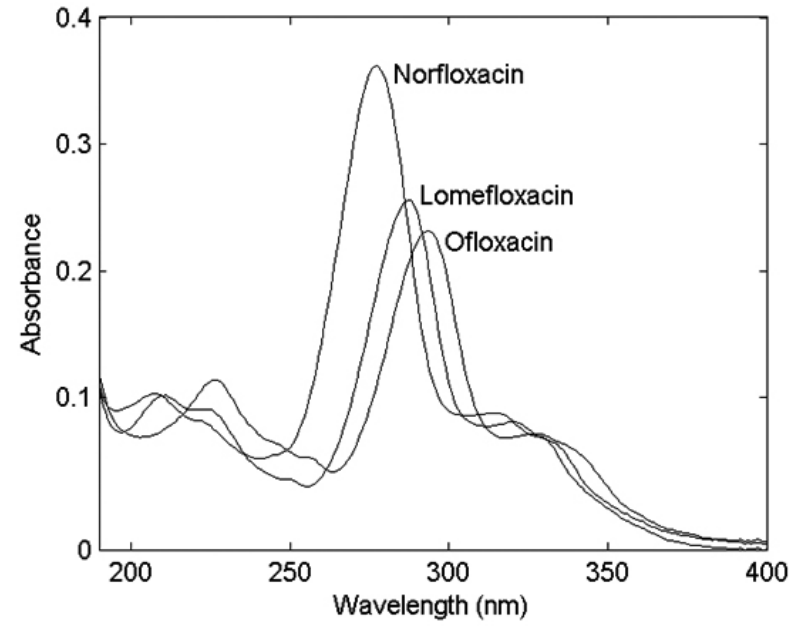

Fig. 2 Absorption spectra of norfloxacin $\left(3.0 \mathrm{mg} \mathrm{L}^{-1}\right)$, ofloxacin $(3.0$ $\left.\mathrm{mg} \mathrm{L}^{-1}\right)$ and lomefloxacin $\left(3.0 \mathrm{mg} \mathrm{L}^{-1}\right)$ in a Britton-Robinson buffer solution $(\mathrm{pH}=1.81)$.

In this paper, a spectrophotometric method is explored for the simultaneous determination of the three important antibacterial agents, norfloxacin, ofloxacin and lomefloxacin. It was based on the spectral differences of the analytes in a Britton-Robinson buffer solution ( $\mathrm{pH}=1.81$ ). The spectral data collected was then processed by use of four chemometric methods including CLS [10-12], PCR, PLS [13-16], and RBF-ANN [17-21]. The prediction performance of the calibration models based on these methods were compared, and the better performing models were applied for the analysis of norfloxacin, ofloxacin and lomefloxacin present in rabbit blood serum.

\section{Theory} $(P L S)$

2.1. Principal component regression $(P C R)$ and partial least square

Principal component regression (PCR) and partial least square (PLS) [1316] are factor analysis multivariate statistical tools which have many of the fullspectrum advantages of the CLS method and have been successfully applied to the analysis of multicomponent mixtures. Like the CLS method, PCR and PLS need a calibration step where the models for the spectra and the component concentrations are deduced from a set of standards, followed by a prediction step in which the concentrations of the unknown are estimated from the sample spectrum. Both of these methods involve spectral decomposition. The PCR decomposition is based entirely on spectral variations without regard for the component concentrations. PCR decomposition is significantly influenced by variations which have no relevance to the analyte concentrations. In PLS, the spectral decomposition is weighted to the concentration. The major difference in the predictive abilities of these two methods is that PLS seems to predict 
better than PCR when there are random linear baselines or independently varying major spectral components which overlap with the spectral features of the analysis.

2.2. Radial basis function-artificial neural network (RBF-ANN)

Recently, the radial basis function-artificial neural network (RBF-ANN) model has been noted for its simple network structure that avoids lengthy calculations as compared to the back propagation -artificial neural network (BP-ANN), and has good robustness, as well as improved sensitivity to noisy data [17]. Typically in an RBF network [17-21], there are three layers: input, hidden and output. The input layer serves only to distribute input to the hidden layer. Each neuron of the hidden layer uses a Gaussian transfer or basis function, instead of the sigmoid one associated with the BP model, to account for the non-linearity. The hidden layer in the RBF network consists of an array of nodes that contain a parameter called the 'radial centre' vector. The hidden layer performs a fixed non-linear transformation with non-adjustable parameters. The approximation of the input-output relationship is derived by obtaining a suitable number of nodes in the hidden layer and by positioning them in the input space where the data is most clustered. For each iteration, the position of the radial centres, their width (variation) and the linear weights are modified for each output node. The learning is completed when each radial centre is located as closely as possible to each discrete cluster centre formed from the input space.

\section{EXPERIMENTAL}

\subsection{Reagents}

Doubly distilled water and analytical-reagent grade chemicals were used throughout the experiments. A stock solution of norfloxacin $\left(60 \mathrm{mg} \mathrm{L}^{-1}\right)$ (Aldrich, Milwaukee, WI, USA), is iomefloxacin $\left(100 \mathrm{mg} \mathrm{L}^{-1}\right)$ (Aldrich, Milwaukee, WI, USA) and lomefloxacin (Aldrich, Milwaukee, WI, USA) were prepared by dissolving the appropriate amount of each compound in a small amount of 0.2 mol L ${ }^{-1}$ sodium hydroxide, and diluted to $500 \mathrm{~mL}$ with demineralised water. A stock solution of $0.2 \mathrm{~mol} \mathrm{~L}^{-1}$ sodium hydroxide was prepared by dissolving $2 \mathrm{~g}$ of sodium hydroxide (The Second Reagent Factory of Shanghai, China) in 250 $\mathrm{mL}$ of water. Britton-Robinson buffer solutions $(\mathrm{pH}=1.81-4.56)$ were prepared by adding different amounts of sodium hydroxide solution $\left(0.2 \mathrm{~mol} \mathrm{~L}^{-1}\right)$ into $100 \mathrm{~mL}$ of mixed acid, containing $0.04 \mathrm{~mol} \mathrm{~L}^{-1}$ of each of boric acid, orthophosphoric acid and acetic acid.

3.2. Apparatus

Spectral data were obtained from measurements on a UV-2450 spectrophotometer (Shimadzu, wavelength width: $190-1100 \mathrm{~nm}$ ) with a 10 $\mathrm{mm}$ pathlength fused-silica cuvette. The $\mathrm{pH}$ was measured with a Model SA $720 \mathrm{pH}$ meter (Orion). The measured data were processed on a Pentium IV computer with CLS, PCR, PLS and RBF programs written in MATLAB 6.5 (Mathworks) by the authors according to some references [14, 18].

3.3. General procedure

Suitable volumes of the mixture of the analytes were transferred to $10 \mathrm{~mL}$ volumetric flasks followed by the addition of $2 \mathrm{~mL}$ of the Britton-Robinson buffer solution $(\mathrm{pH}=1.81)$, diluted to the mark with water and mixed well. An absorption spectrum was measured for each solution between 190 and $400 \mathrm{~nm}$ at $1 \mathrm{~nm}$ interval in $10 \mathrm{~mm}$ cuvettes with respect to a reagent blank.

3.4. Procedure for the determination of norfloxacin, ofloxacin and lomefloxacin in rabbit blood serum

A certain volume of acetonitrile was added to $0.2 \mathrm{~mL}$ rabbit blood serum containing a certain amount of norfloxacin, ofloxacin and lomefloxacin in a $1.5 \mathrm{~mL}$ micro-centrifuge tube. The samples were centrifuged at $56000 \mathrm{~g}$ for $30 \mathrm{~min}$. The supernatant solution was transferred to a $5 \mathrm{~mL}$ ampoule and the acetonitrile removed by extraction with $1 \mathrm{~mL}$ dichloromethane. The sample was centrifuged at $5600 \mathrm{~g}$ for $8 \mathrm{~min}$, and the aqueous supernatant transferred to a sample tube. Then, $500 \mu \mathrm{L}$ aliquot of this solution was analyzed following the above mentioned General Procedure (Section 3.3).

\section{RESULTS AND DISCUSSION}

4.1. Spectral behaviour of norfloxacin, ofloxacin and lomefloxacin

The absorption spectra $(190-400 \mathrm{~nm})$ of norfloxacin, ofloxacin and lomefloxacin in a Britton-Robinson buffer solution ( $\mathrm{pH}=1.81$ ) (Fig. 2) showed that they overlapped significantly, and the absorbance maxima were $277 \mathrm{~nm}$ for norfloxacin, $293 \mathrm{~nm}$ for ofloxacin, and $287 \mathrm{~nm}$ for lomefloxacin, respectively. This precluded the application of traditional spectrophotometric approaches for the simultaneous determination of the analytes, and thus, the use of the chemometric methods based on spectral resolution was available.

4.2. Effect of $p H$ on the absorbance of norfloxacin, ofloxacin and lomefloxacin

The effect of $\mathrm{pH}$ of Britton-Robinson buffer solutions in the range of $1.81-11.98$ was studied. In general, it was found that the peak intensities at absorption maxima decreased with the increase of $\mathrm{pH}$. Thus, a $\mathrm{pH}=1.81$ Britton-Robinson buffer solution was selected for all studies in this work.

4.3. Calibration curves and limits of detection

Calibration spectra of the norfloxacin, ofloxacin and lomefloxacin solutions (Fig. 3), run under the proposed experimental conditions, were linearly modeled, and the figures of merit for the three compounds were calculated (Table 1). The linear concentration range is $0.6-13.8 \mathrm{mg} \mathrm{L}^{-1}$ for norfloxacin, $0.5-15.0$ $\mathrm{mg} \mathrm{L}^{-1}$ for ofloxacin and $0.5-15.0 \mathrm{mg} \mathrm{L}^{-1}$ for lomefloxacin, respectively. LOD value is $0.14 \mathrm{mg} \mathrm{L}^{-1}$ for norfloxacin, $0.24 \mathrm{mg} \mathrm{L}^{-1}$ for ofloxacin and $0.06 \mathrm{mg}$ $\mathrm{L}^{-1}$ for lomefloxacin, respectively. Both the above linear ranges and the LODs compare well with the figures of merit obtained with the HPLC method, which utilize a UV detector [4].
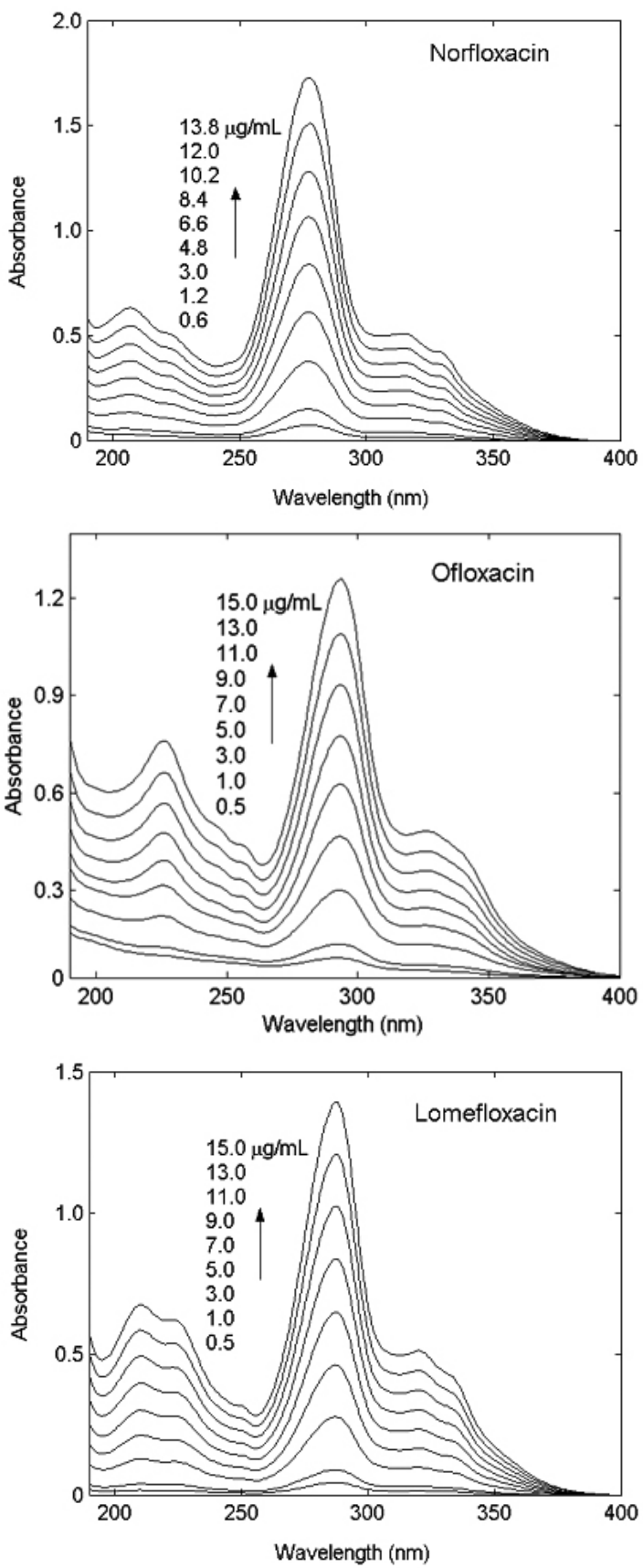

Fig. 3 Absorption spectra of norfloxacin, ofloxacin and lomefloxacin with different concentrations in a Britton-Robinson buffer solution $(\mathrm{pH}=1.81)$. 
Table 1 .- Comparison of analytical figures of merit for the determination of norfloxacin, ofloxacin and lomefloxacin

\begin{tabular}{lllllllll}
\hline & $\begin{array}{l}\text { Number of } \\
\text { samples }(\mathrm{n})\end{array}$ & $\begin{array}{l}\text { Linear range } \\
\left(\mathrm{mg} \mathrm{L}^{-1}\right)\end{array}$ & $\begin{array}{l}\text { Correlation } \\
\text { coeffcient }\end{array}$ & $\begin{array}{l}\text { Intercept } \\
\left(\times 10^{-3}\right)\end{array}$ & $\begin{array}{l}\text { Slope } \\
\left(\mathrm{L} \mathrm{mg}^{-1}\right)\end{array}$ & $\begin{array}{l}\mathrm{s}_{\mathrm{i}}^{\mathrm{a}} \\
\left(\times 10^{-3}\right)\end{array}$ & $\begin{array}{l}\mathrm{s}_{\mathrm{s}}^{\mathrm{a}} \\
\left(\times 10^{-3}\right)\end{array}$ & $\begin{array}{l}\mathrm{LOD}^{\mathrm{a}} \\
\left(\mathrm{mg} \mathrm{L}^{-1}\right)\end{array}$ \\
\hline $\begin{array}{l}\text { Norfloxacin } \\
\text { Ofloxacin }\end{array}$ & 9 & $0.6-13.8$ & 0.9999 & 1.6 & 0.126 & 3.6 & 0.45 & 0.14 \\
Lomefloxacin & 9 & $0.5-15.0$ & 0.9999 & 57.6 & 0.08 & 3.7 & 0.43 & 0.24 \\
\hline
\end{tabular}

${ }^{a} s_{\mathrm{i}}, \mathrm{s}_{\mathrm{s}}$ and LOD are the standard deviation of the intercept, the standard deviation of the slope and the limits of detection, respectively.

4.4. Chemometric analysis of synthetic mixtures of norfloxacin, ofloxacin and lomefloxacin

The composition of the calibration sets (total - 12 standards, Table 2) was orthogonally designed, according to a three-level orthogonal array [22], denoted by $\mathrm{OA}_{9}\left(3^{4}\right)$, in order to obtain maximum information from the calibration procedure. The range of concentrations used for norfloxacin, ofloxacin and lomefloxacin were $0.6-10.2,0.5-6.0$ and $0.5-6.0 \mathrm{mg} \mathrm{L}^{-1}$, respectively. Spectra of these samples were collected according to the experimental procedure described above, which was used with the aid of chemometric methods such as CLS, PCR, PLS, and RBF-ANN. Outliers in spectral data measured in all samples (the calibration sets plus the following validation sets) can be detected by the Q-statistics [23]. The Q-statistics is a measure of the difference of a sample and its projection onto the principal component analysis (PCA) model and high Q-values indicate extreme samples that are not very well modeled. The results showed that outliers in spectral data measured in all samples did not exist [23].

To avoid overfitting or underfitting in PCR, PLS and RBF models, the cross-validation procedures were employed and submitted to the calibration sets. The cross-validation procedures consisting of systematically removing one of the calibration samples in turn, and using only the remaining ones for the construction of the parameters and regression were applied [24].

The predicted concentrations were then compared with the known ones for each of the calibration samples and the predicted error sum of squares (PRESS) was calculated. The PRESS was computed in the same manner each time a new parameter was added to the models. PRESS was calculated according to Eq. (1):

$$
\operatorname{PRESS}=\sum_{\mathrm{i}=1}^{\mathrm{n}}\left(\mathrm{c}_{\mathrm{ij}(\text { found })}-\mathrm{c}_{\mathrm{ij} \text { (added) }}\right)^{2}(1)
$$

where $c_{i j \text {,added }}$ indicates the concentration of $j$ th component in $i$ th mixtures in the calibration set, $c_{i j \text { pred }}$ is its estimation in the calibration set found by mentioned above chemometrics methods.

One reasonable choice for the optimum parameters would be the number, which yields the minimum PRESS. The results showed that the maximum number of factors used to calculate the optimum PRESS with the aid of PCR and PLS methods was 3 and 4, and the parameters of nodes in the hidden layer and the spread coefficient in the RBF method are 5 and 163 , respectively.

To evaluate the prediction performance of these chemometric models, a set of nine synthetic validation mixtures were prepared (Table 3), and submitted for prediction by each of the calibration models. The prediction ability was expressed in terms of the relative prediction errors- $-\mathrm{RPE}_{\mathrm{S}}$ for individual compounds, and $\mathrm{RPE}_{\mathrm{T}}$ for the overall error (Table 4).

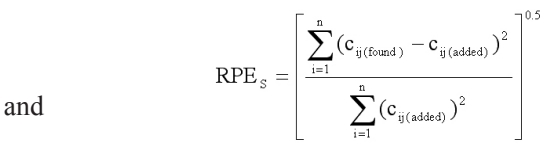

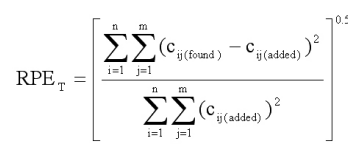

where $c_{i j, \text { added }}$ is the concentration of $j$ th component in $i$ th mixtures, and $c_{i j}$ is its estimation found by the above mentioned chemometric methods [25].

From Table 4 , it can be seen that $\% \mathrm{RPE}_{\mathrm{S}}$ and $\% \mathrm{RPE}_{\mathrm{T}}$ given by the PCR model were better than those obtained by the CLS model, and slightly worse than those obtained by PLS model. This reflects the usual experience with these three methods. In addition, the RBF-ANN one produced better results than the PLS model on the basis of the $\% \mathrm{RPE}_{\mathrm{S}}$ and $\% \mathrm{RPE}_{\mathrm{T}}$ criterion. This is because the RBF-ANN are also effective for modeling non-linear signal-property relationships. Therefore, the RBF-ANN is selected for the simultaneous determination of norfloxacin, ofloxacin and lomefloxacin in mixtures based on low $\% \mathrm{RPE}_{\mathrm{S}}$ and $\% \mathrm{RPE}_{\mathrm{T}}$

Table 2.- Composition of the calibration samples

\begin{tabular}{llll}
\hline Sample & \multicolumn{3}{c}{ Concentration $\left(\mathrm{mg} \mathrm{L}^{-1}\right)$} \\
\hline & Norfloxacin & Ofloxacin & Lomefloxacin \\
& & & \\
1 & 5.4 & 0.5 & 10.0 \\
2 & 0.6 & 10.0 & 10.0 \\
3 & 10.2 & 5.0 & 10.0 \\
4 & 0.6 & 0.5 & 0.5 \\
5 & 10.2 & 10.0 & 0.5 \\
6 & 5.4 & 5.0 & 0.5 \\
7 & 10.2 & 0.5 & 5.0 \\
8 & 5.4 & 10.0 & 5.0 \\
9 & 0.6 & 5.0 & 5.0 \\
10 & 0.0 & 0.0 & 6.0 \\
11 & 0.0 & 6.0 & 0.0 \\
12 & 7.2 & 0.0 & 0.0
\end{tabular}

Table 3.- Composition of the validation samples

\begin{tabular}{clll}
\hline Sample & \multicolumn{3}{c}{ Concentration $\left(\mathrm{mg} \mathrm{L}^{-1}\right)$} \\
\hline & Norfloxacin & Ofloxacin & Lomefloxacin \\
\hline 1 & 1.2 & 1.0 & 4.0 \\
2 & 9.6 & 4.0 & 4.0 \\
3 & 6.0 & 8.0 & 4.0 \\
4 & 9.6 & 1.0 & 8.0 \\
5 & 6.0 & 4.0 & 8.0 \\
6 & 1.2 & 8.0 & 8.0 \\
7 & 6.0 & 1.0 & 1.0 \\
8 & 1.2 & 4.0 & 1.0 \\
9 & 9.6 & 8.0 & 1.0 \\
\hline
\end{tabular}

Table 4.- Comparison of prediction results for the synthetic samples with the aid of different chemometric methods

\begin{tabular}{lllll}
\hline \multicolumn{2}{c}{ Chemometric methods $\% \mathrm{RPE}_{\mathrm{S}}$} & \multirow{2}{*}{ RPPE $_{\mathrm{T}}$} \\
\cline { 2 - 5 } & Norfloxacin & Ofloxacin & Lomefloxacin & \\
\hline CLS & $3.2(92.4)^{\mathrm{a}}$ & $1.6(78.6)$ & $11.1(124.6)$ & 8.8 \\
PCR $^{\mathrm{b}}$ & $0.7(100.4)$ & $5.1(88.3)$ & $12.2(125.8)$ & 7.0 \\
PLS $^{\mathrm{c}}$ & $0.7(100.4)$ & $5.0(88.4)$ & $12.1(125.7)$ & 6.9 \\
RBF-ANN $^{\mathrm{d}}$ & $1.3(108.0)$ & $2.22(96.1)$ & $4.91(108.0)$ & 3.0 \\
\hline
\end{tabular}

a Values in the parentheses correspond to the mean recoveries (\%).

${ }^{\mathrm{b}}$ The number of factors used with use of PCR method is 3 .

c The number of factors used with use of PLS method is 4 .

${ }^{\mathrm{d}}$ Data pretreatment with normalization. The parameters of nodes in the hidden layer and the spread coefficient are 5 and 163 , respectively. 


\subsection{Interfering effects}

In order to evaluate the practical applicability of the present procedure for the simultaneous determination of norfloxacin, ofloxacin and lomefloxacin in real samples, the potential interfering effects of some species frequently encountered in rabbit blood serum were investigated by analyzing a standard solution containing $5.0 \mathrm{mg} \mathrm{L}^{-1}$ norfloxacin, $5.0 \mathrm{mg} \mathrm{L}^{-1}$ ofloxacin, $5.0 \mathrm{mg} \mathrm{L}^{-1}$ lomefloxacin and various amounts of coexisting species. The concentration ratios of foreign species to the standard solution, which are tolerated within a $\pm 10 \%$ error range, are as follows: $\mathrm{Cd}(\mathrm{II}), \mathrm{Na}(\mathrm{I}), \mathrm{K}(\mathrm{I}), \mathrm{Mg}(\mathrm{II}), \mathrm{SO}_{4}{ }^{2-}, \mathrm{Cl}^{-}$and glucose (300), tartrate (100), $\mathrm{Co}(\mathrm{II}), \mathrm{Fe}(\mathrm{II}), \mathrm{Cu}(\mathrm{II})$ and $\mathrm{Zn}$ (II) (30), ascorbic acid (5).

4.6. Determination of norfloxacin, ofloxacin and lomefloxacin in rabbit blood serum

The proposed method for the simultaneous determination of norfloxacin, ofloxacin and lomefloxacin with the aid of RBF-ANN model, was applied for the analysis of the rabbit blood serum. The results are summarized in Table 5. The results obtained by the proposed method were very close to those obtained by HPLC. The relative standard errors between the proposed method and HPLC are below $10 \%$. This indicated that there was no significant difference between the proposed method and the reference method. Consequently, the above findings indicate that proposed method can be successfully applied for simultaneous determination of norfloxacin, ofloxacin and lomefloxacin in real samples

Table 5.- Determination of norfloxacin, ofloxacin and lomefloxacin (mg/g) in rabbit blood serum with the use of RBF-ANN model, and by the alternative technique HPLC

\begin{tabular}{llll}
\hline Method & Norfloxacin & Ofloxacin & Lomefloxacin \\
\hline RBF-ANN & 1.31 & 1.08 & 0.75 \\
HPLC & 1.28 & 1.13 & 0.79 \\
\%RSE & 2.3 & -4.4 & -5.1 \\
\hline
\end{tabular}

${ }^{a}$ Percent relative standard error between RBF-ANN and HPLC.

\section{CONCLUSION}

A UV-spectrophotometric method of analysis was successfully researched and developed for the simultaneous determination of norfloxacin, ofloxacin and lomefloxacin commonly found in rabbit blood serum. Multivariate calibration models were built from the spectral data matrices on the basis of CLS, PCR, PLS and RBF-ANN methods. Verification of the calibrations carried out with the aid of a synthetic set of mixtures of the three compounds, produced satisfactory results on the basis of figures of merit with the method comparing well with HPLC in the literature. The results also showed that for practical purposes CLS models performed poorly in comparison with the PCR, the PLS and the RBF-ANN ones, and the RBF-ANN is the best model in this system. Thus, the RBF-ANN model was employed for analysis of the three compounds in commonly available rabbit blood serum.

\section{ACKNOWLEDGEMENTS}

The authors gratefully acknowledge the financial support of this study by the Jiangxi Province Natural Science Foundation (JXNSF[2006]144).

\section{REFERENCES}

[1] A.R. Martin in Textbook of Organic Medicinal and Pharmaceutical Chemistry, J.N. Delgado, W.A. Remers eds. J.B. Lippincott Company, 1991.

[2] D.C. Hooper, J.S. Wolfson, N. Engl, J. Med. 324, 384, (1991).

[3] E. Debbia, G. C. Schito, G. Nicoletti, A. Speciale, Chemotherapy 6, 313, (1987).

[4] G.H. Guo, W. Pan, D.S. Su, H.Z. Chen, Chinese J. Chromatogr. 23, 401, (2005).

[5] P.A. Dagostino, J.R. Hancock, L.R. Provost, Rapid Commu. Mass Spectrom. 9, 1038, (1995).

[6] R.C. Barthus, L.H. Mazo, R.J. Poppi, J. Pharm. Biomed. Anal. 38, 94, (2005).

[7] S.W. Li, J.Z. Pan, Y. He, X.H. Wang, Chem. Res. Appl. 17, 471, (2005).

[8] P . Evans, K.C. Persaud, A.S. McNeish, Sensor Actuat. A, 69, 348, (2000).

[9] S. Sadeghhi, E. Karimi, Chem. Pharm. Bull. 54, 1107, (2006).

[10] M.K. Antoon, J.H. Koenig, E.L. Koenig, Appl. Spectrosc. 31, 518, (1977).

[11] D.M. Haaland, R.G. Easterling, Appl. Spectrosc. 34, 539, (1980)

[12] D.M. Haaland, R.G. Easterling, Appl. Spectrosc. 36, 65, (1982).

[13] I.T. Jolliffe, Principal Component Analysis, Springer, New York, 1986.

[14] W. Lindberg, J.A. Persson, S. Word, Anal. Chem. 55, 643, (1983).

[15] A. Lorber, L.E. Wangen, B.R. Kowalski, J. Chemometr. 1, 19, (1987).

[16] Y.N. Ni, Y. Wang, S. Kokot, Anal. Lett. 40, 1029, (2007).

[17] A. Pulido, I. Ruisanchez, F.X. Rius, Anal. Chim. Acta, 388 (1999) 273281

[18] Y.N. Ni, Y. Wang, Microchem. J. 86, 216, (2007).

[19] M. Blanco, S. Maspoch, I. Villarroya, X. Peralta, J. M. Gonzalez, J. Torres, Analyst 126, 378, (2001).

[20] F. Despagne, D. L. Massart, Analyst 123, 157R, (1998).

[21] A. Pulido, I. Ruisanchez, F. X. Rius, Anal. Chim. Acta 388, 273, (1999).

[22] W.G. Lan, M.K. Wong, N. Chen, Y.M. Sin, Analyst 119, 1669, (1994).

[23] Eigenvector Research, Inc., 830 Wapato Lake Road, Manson, WA 98831, US.

[24] R.G. Brereton, Chemometrics: Data Analysis for the Laboratory and Chemical Plant, Wiley, Chichester, 2003.

[25] Y.N. Ni, Y.R. Wang, S. Kokot, Talanta 69, 216, (2006). 